\title{
Application of Simple Random Sampling in Agriculture using R-software
}

\author{
M. Iqbal Jeelani ${ }^{1 *}$, Nageena Nazir ${ }^{1}$, S. A. Mir ${ }^{1}$, Fehim Jeelani ${ }^{1}$, N. A. Dar ${ }^{2}$, \\ Shamsul $\mathrm{Haq}^{3}$, Syed $\mathrm{Maqbool}^{3}$, Shahid wani ${ }^{3}$ \\ 'Division of Agricultural Statistics, SKUAST-K, India; jeelani.miqbal@gmail.com \\ 2Division of Plant Pathology, SKUAST-K, India \\ ${ }^{3}$ Division of Environmental Science, SKUAST-K, India
}

\begin{abstract}
In this paper one of the basic techniques of sampling namely simple random sampling has been used utilizing R software keeping in view the importance of this technique in agricultural surveys. Apple data taken from district Ganderbal of Kashmir valley is taken. Function SRSWOR(Y,N) using R software is developed; also different graphics are presented.
\end{abstract}

Keywords: R Software, Boxplot, Simple Random Sampling

\section{Introduction}

Sample surveys are widely used as a means of collecting information to meet a definite need in agriculture, trade, social, educational and economical problems. It has been observed that the sample survey can give very precise information. Since in a sample survey, a part of population is surveyed and inference is drawn about the whole population, the results are subject to be different from the true population values, but the advantage with the sample survey is that this type of error can be measured and controlled. Sample survey is less time consuming, involves less cost, has greater scope in special coverages and also has greater operational facilities as compared to complete enumeration. It is for these reasons that sample surveys are being preferred and adopted frequently by the government, scientific organizations, industries, institutes and others since the beginning of $20^{\text {th }}$ century. One of the vital issues in a sample survey is the choice of proper sampling techniques. In the choice of a sampling method, there are some methods of selection while others are control measures which help in grouping the population before the selection process. Simple random sampling is one of the important technique employed in sample survey. Simple random sampling is method of selecting $n$ units out of a population of size $N$ by giving equal probability to all units, or sampling procedure in which all possible combinations of $n$ units that may be formed from the population of $N$ units have the same probability of selection. It is also sometimes referred as unrestricted random sampling. If a unit is selected and noted and then returned back to the population before the next drawing is made and this procedure repeated $n$ times, it gives rise to a simple random sample of $n$ units. This procedure is generally known as simple random sampling with replacement (srswr). If this procedure is repeated till $n$ distinct units are selected and all repetitions are ignored. It is called a simple random sampling without replacement (srswor). This implies that in srswor from a population of size $N$, the probability that any sampling unit is included in the sample is $1 / N$ and this probability remains constant throughout the drawing. Mathematically if $E_{\mathrm{r}}$ is the event that any specified unit is selected at the $r^{\text {th }}$ draw, $P\left(E_{\mathrm{r}}\right)=$ $1 / \mathrm{N},(r=1,2,3, \ldots \ldots \ldots, n)$, where $\mathrm{n}$ is the sample size. In particular it implies $P\left(E_{\mathrm{r}}\right)=1 / \mathrm{N}=\mathrm{P}\left(E_{1}\right)$ i.e., the chance of selection of any specified item is same at any draw as it was in the first draw, viz., $1 / \mathrm{N}$

\section{Material Methods}

In simple random sampling without replacement (srswor), the variance of the sample mean is given by;

${ }^{*}$ Author for correspondence 
$(\bar{x})_{s r s w o r}=\frac{N-n}{N} \frac{S^{2}}{n}$. The standard error of $\bar{x}$ is given by; $S . E(\bar{x})=\sqrt{\left(\frac{N-n}{N}\right) \frac{S^{2}}{n}}=\sqrt{\left(1-\frac{n}{N}\right)} \cdot \frac{S}{\sqrt{n}}$. The factor $n / N$ is called sampling fraction and the factor $\frac{N-n}{N}=\left(1-\frac{n}{N}\right)$ is called the finite population correction (f.p.c) factor. If the population size is very large or we have an infinite population or if $\mathrm{n}$ is relatively very small compared with $\mathrm{N}$, then $n / N \rightarrow 0=>\left(1-\frac{n}{N}\right) \rightarrow 1$. Thus, for an infinite population (i.e., $N \rightarrow \infty$ ), then $\operatorname{var}(\bar{x})_{s r s w r}=\frac{N-n}{N} \frac{S^{2}}{n}=\frac{S^{2}}{n}$. Hence we have $\operatorname{var}(\bar{x})=\frac{\sigma^{2}}{n} \Rightarrow \operatorname{S.E}(\bar{x})=\frac{\sigma}{\sqrt{n}}$, which is very important result in sampling theory ${ }^{1}$.

Simple random sampling with replacement (srswr) can be regarded as sampling from an infinite population and its variance is given by; $\operatorname{var}(\bar{x})_{s r s w r}=\frac{N-1}{N} \frac{S^{2}}{n}$. Comparing the variance of srswr with srswor, we get $\operatorname{var}(\bar{x})_{\text {srswr }}<\operatorname{var}(\bar{x})_{\text {srswor }}$ i.e., the variance of sample mean is less in srswor as compared with its variance in the case of srswr. This implies that srswor provides a better (more efficient) estimate of the population mean $\mu$ relative to srswr. The following notations shall be used in discussion that follows;

$\mathrm{N}=$ size of the population

$\mathrm{y}=$ value of the characteristic under study

$\bar{y}=\frac{1}{N} \sum_{i=1}^{N} y_{i}=$ Population mean

$S^{2}=\frac{1}{N-1} \sum_{i=1}^{N}\left(y_{i}-\bar{y}\right)^{2}=$ Population mean square

$\bar{y}_{n}=\frac{1}{n} \sum_{i=1}^{n} y_{i}=$ Sample mean

$s^{2}=\frac{1}{n-1} \sum_{i=1}^{n}\left(y_{i}-\bar{y}_{n}\right)^{2}=$ Sample mean square

$V\left(\bar{y}_{n}\right)=\frac{N-n}{N n} S^{2}=$ Variance

$\operatorname{S.E}\left(\bar{y}_{n}\right)=\frac{\sqrt{V\left(\bar{y}_{n}\right)}}{\sqrt{(1-f) S^{2} / n}}$

The block Ganderbal was selected for the present study in District Ganderbal. District Ganderbal being inseparable part of the state naturally inherits the same characteristics which predominately exist in the economy of the state. Agriculture is the main source of income and employment in the district. More than half of the population, directly and indirectly derive their livelihood from it. Paddy, maize and horticulture are the principle crops grown in the district. There is good network of agricultural infrastructure available throughout the length and breadth of the district. Total area sown under different food and non-food crops is about 27735 hectares, out of which 15828 hectares constituting 57 per cent was under cereal food crops. At present 8738 hectares are under major horticulture crops with 3866 hectares constituting 44 per cent are under apple cultivation and out of 47916 MT of production of horticulture crops, apple production is $34873 \mathrm{MT}$ which is 72 per cent of the total production.

A survey was conducted for estimation of average yield in the district Ganderbal at block level. Since at present 8738 hectares are under major horticulture crops with 3866 hectares constituting 44 percent of the area is under apple cultivation in district Ganderbal. A total of 420 orchards were reported in the block Ganderbal covering an area of 772.8 hectares with 73,496 total number of trees. Total production of apple in the block was found out to be 6758.52 metric tons (Mt) with the productivity of $8.74 \mathrm{Mt} / \mathrm{ha}$. American, Delicious and Maharaji were the main varieties of apple cultivated in the block.

\subsection{Utilization of R-software in Sample Survey Data}

$\mathrm{R}$ software is a system for graphics and statistical analyses developed by the AT\&T Bell Laboratories USA . Its development and distribution is being carried out by several well known statisticians commonly known as the $\mathrm{R}$ Development Core Team ${ }^{5}$. Comprehensive R Archive Network (CRAN) is the core internet site from which files needed to install $\mathrm{R}$ are being used. $\mathrm{R}$ has many functions usually visualized in their own window and are saved in several formats like jpg, png, bmp, ps, pdf, emf, pictex and xfig. The results from a statistical analysis are displayed on the screen; some intermediate results (P-values, regression coefficients, residuals, etc) can be saved, written in a file, or used in subsequent analyses. It is also possible to combine in single program different statistical functions to perform more complex analyses. At first, R could seem too complex for a non-specialist. This may not be true actually. In fact, a prominent feature of $\mathrm{R}$ is its flexibility. Whereas classical software displays 
immediately the results of an analysis, $\mathrm{R}$ stores these results in an object, so that an analysis can be done with no result displayed. The user may be surprised by this, but such a feature is very useful. The user can extract only the part of the results which is of interest. Various versions of R-software are available on web site http:// cran.r-project.org. Some of the important books written by Becker et al. $^{2}$ and Venables and Repley ${ }^{3}$ are of immense use for understanding the main features of this software. Khan and Mir ${ }^{4}$ have discussed in detail the application of R-software in agricultural sciences.

\section{Preliminary Study of Data Structure}

To have a comprehensive look into the data its graphic as well as numerical summary is required. This job can be accomplished using the functions of $\mathrm{R}$ which are specially meant for meeting this requirement.

\section{Graphical summary}

A graphical summary of the data set provides its visible picture which can be easily understood by a common man.

Boxplot() To get the box plot of Yield, Area, Trees, Bearing and Non-Bearing trees per orchard of apple data the commands are given below; op $=$ par $(\mathrm{mfrow}=$ $c(2,3))$

\# par can be used to set or query graphical parameters. Parameters can be set by specifying them as arguments to par in tag = value form, or by passing them as a list of tagged values.

boxplot(apple\$Yield,ylab="Mt/orchard", main=" Yield")

boxplot(apple \$Area,ylab="ha/orchard",main="Area") boxplot(apple\$Trees,ylab="no/orchard", main=" Trees")

boxplot(apple\$Bearing,ylab="no/orchard",main=" Bearing")

boxplot (apple\$NonBearing,ylab="no/orchard", main="Non-Bearing")

\section{R-function in Simple Random Sampling}

To draw inference about the estimate, standard error, estimated variance and confidence interval of simple random sampling without replacement, the function SRSWOR1(Y,N)is implemented. It takes the argument $\mathrm{Y}$ (data vector) and $\mathrm{N}$ (size of the population) and returns estimates, confidence interval, estimated variance and standard error with a probability content of .95 . The code of this function is

$>$ SRSWOR1<-function $(\mathrm{Y}, \mathrm{N})$

\# A function for estimation in simple random sampling without replacement. This function returns estimates with standard error and $95 \mathrm{~s}$ confidence interval. It requires only data vector and N.Y is the data vector of responses on $\mathrm{n}$ units sampled. $\mathrm{N}$ is the population size \#
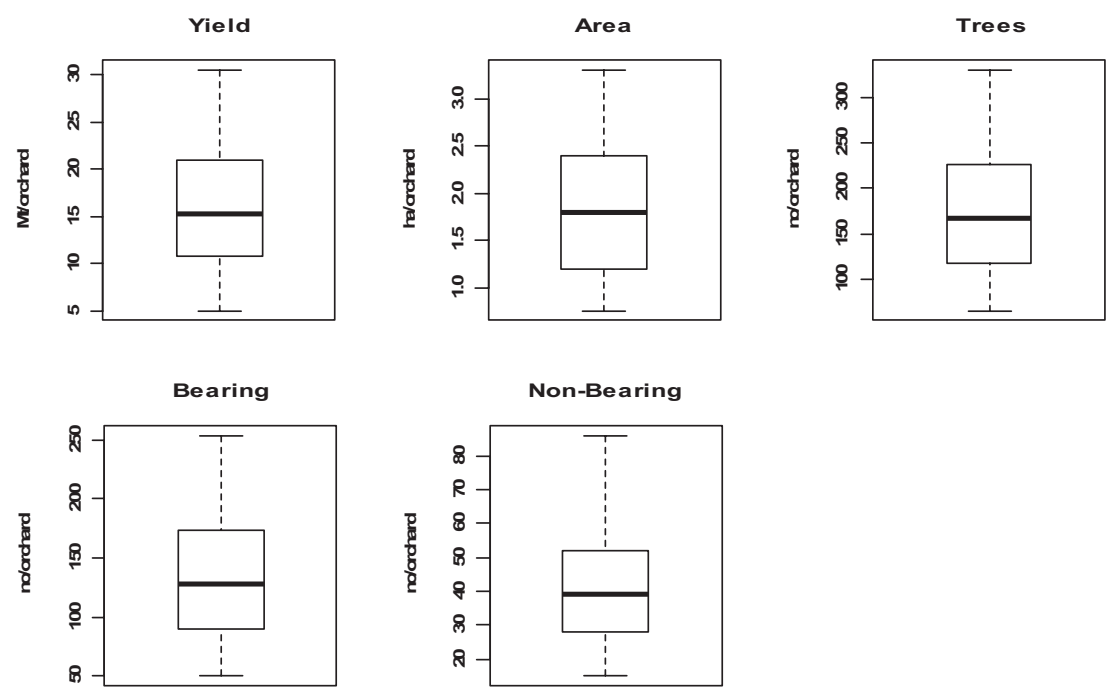

Figure 1. Box plot of Yield, Area, Trees, Bearing and Non-Bearing trees per orchard of apple data. 
Table1. Summary statistics of yield, area and number of trees

\begin{tabular}{lrrrrr}
\hline Variable & Estimate & Standard error & Variance & \multicolumn{2}{c}{ 95\% confidence interval } \\
\cline { 5 - 6 } & & & & Lower limit & Upper Limit \\
\hline Yield (mt) & 14.35 & 0.73 & 37.83 & 13.11 & 15.99 \\
Area (ha) & 1.65 & 0.08 & 0.45 & 1.50 & 1.82 \\
Trees (No.) & 158.00 & 7.72 & 4400.11 & 142.64 & 173.72 \\
\hline
\end{tabular}

\section{\{}

$\mathrm{n}<$-length $(\mathrm{Y})$

Ybar $<$-mean $(\mathrm{Y})$

$\mathrm{S} 2<-\operatorname{var}(\mathrm{Y})$

Estvar $<-\left((\mathrm{N}-\mathrm{n}) /\left(\mathrm{N}^{*} \mathrm{n}\right)\right)^{*} \mathrm{~S} 2$

SeEst $<-$ sqrt(Estvar)

ci<-c(Ybar-1.96*SeEst,Ybar+1.96* SeEst)

$\mathrm{ci}<-\mathrm{c}(\mathrm{ci}[1], \mathrm{Ybar}, \mathrm{ci}[2])$

names(ci)<-c("lower'”'estimate'"'upper")

out $1<-c($ Estimate $=$ Ybar,SE=SeEst $)$

out $1<$-round(out 1,3$)$

out $2<-$ ci

out3=round(out2,3)

list (estimates=out 1,"95\% cont.interval" $=$ out 3 , mean $=Y$

bar,variance $=$ S2, estimatedvariance $=$ Estvar,standarderror $=$ SeEst)

\}

A sample size of 60 orchards was taken from a population 420 orchards. The function SRSWOR1 (Y, N)was run on sample of 60 orchards taken from each variable (yield, area and trees). The results of SRSWOR (60 sample size, Yield, Area, Trees) are given in Table 1.

\section{Conclusion}

It is concluded that simple random sampling procedure is more attractive than its counter parts as they are less time consuming and operationally more convenient.
It has been established that simple random sampling has its practical implications. It can be applied to analyze data generated in a scientific investigation. R-software package facilitates a lot in implementation of simple random sampling techniques which are very informative and applicable to sample surveys of horticultural crops.

\section{Aknowledgement}

The first author wishes to record his gratitude and thanks to university grants commission, Govt. of India for providing the UGC national fellowship for persuing the Doctorate program in Statistics.

\section{References}

1. Cochran WG. Sampling Techniques. New York: John Wiley and Sons; 1977.

2. Becker RA, Chambers JM, Wilks AR. The New S Language. New York: Chapman and Hall; 1988.

3. Venables WN, Ripley BD. Modern Applied Statistics with S-PLUS, 4th ed. New York: Springer Verlag; 2009.

4. Khan AA and Mir AH. Applications of R- software in agriculturaldataanalysis.SKUASTJournalofResearch. 2005;7(1): 36-64.

5. R Development Core Team. A language and an environmentforstatisticalcomputing. Vienna, Austria:RFoundation forStatisticalComputing;1995. Availablefromhttp://CRAN.RProject.org/ 